\title{
Carotid Plaque Morphology and Ischemic Vascular Brain Disease on MRI
}

\author{
(D).J.A. van den Bouwhuijsen, (D) M.W. Vernooij, DB.F.J. Verhaaren, DH.A. Vrooman, (D)W.J. Niessen, (D) G.P. Krestin, (D) M.A. Ikram, \\ DO.H. Franco, and (D) A. van der Lugt
}

\begin{abstract}
BACKGROUND AND PURPOSE: Vulnerable carotid plaque components are reported to increase the risk of cerebrovascular events. Yet, the relation between plaque composition and subclinical ischemic brain disease is not known. We studied, in the general population, the association between carotid atherosclerotic plaque characteristics and ischemic brain disease on MR imaging.
\end{abstract}

MATERIALS AND METHODS: From the population-based Rotterdam Study, 951 participants underwent both carotid MR imaging and brain MR imaging. The presence of intraplaque hemorrhage, lipid core, and calcification and measures of plaque size was assessed in both carotid arteries. The presence of plaque characteristics in relation to lacunar and cortical infarcts and white matter lesion volume was investigated and adjusted for cardiovascular risk factors. Stratified analyses were conducted to explore effect modification by sex. Additional analyses were conducted per carotid artery in relation to vascular brain disease in the ipsilateral hemisphere.

RESULTS: Carotid intraplaque hemorrhage was significantly associated with the presence of cortical infarcts (OR, 1.9; 95\% confidence interval, 1.1-3.3). None of the plaque characteristics were related to the presence of lacunar infarcts. Calcification was the only characteristic that was associated with higher white matter lesion volume. There was no significant interaction by sex.

CONCLUSIONS: The presence of carotid intraplaque hemorrhage on MR imaging is independently associated with MR imaging-defined cortical infarcts, but not with lacunar infarcts. Plaque calcification, but not vulnerable plaque components, is related to white matter lesion volume.

ABBREVIATIONS: $\mathrm{PD}=$ proton density; $\mathrm{WML}=$ white matter lesion

dentification of individuals who are at high risk for cerebrovascular events is an important goal in the prevention of cerebrovascular disease. There is growing awareness that the risk of a cerebrovascular event depends on not only the severity of carotid stenosis but also the morphology and composition of the athero-

Received April 6, 2017; accepted April 27.

From the Departments of Epidemiology (Q.J.A.v.d.B., M.W.V., B.F.J.V., M.A.I., O.H.F.), Radiology (Q.J.A.v.d.B., M.W.V., H.A.V., W.J.N., G.P.K., M.A.I., A.v.d.L.), and Medical Informatics (H.A.V., W.J.N.), Erasmus MC, Rotterdam, the Netherlands.

The Rotterdam Study is funded by Erasmus Medical Center and Erasmus University Rotterdam, the Netherlands; the Research Institute for Diseases in the Elderly; the Ministry of Education, Culture and Science; the Ministry for Health, Welfare and Sports; the European Commission (DG XII); and the Municipality of Rotterdam. This study was supported by grants from the Netherlands (Dutch) Heart Foundation (2006B206, 2009B044, and 2009B102), the Netherlands Organization for Scientific Research/the Netherlands Organization for Health Research and Development ZonMw (Vici grant 918.76.619), and the Netherlands Consortium for Healthy Ageing.

Please address correspondence to Aad van der Lugt, MD, PhD, 's-Gravendijkwal 230, PO Box 2040, 3000CA Rotterdam, the Netherlands; e-mail: a.vanderlugt@erasmusmc.nl

-- Indicates open access to non-subscribers at www.ajnr.org

= Indicates article with supplemental on-line tables.

http://dx.doi.org/10.3174/ajnr.A5288 sclerotic plaque. ${ }^{1,2}$ Histologic studies indicate that specific "vulnerable" plaque characteristics, such as a large lipid core, thin fibrous cap, and intraplaque hemorrhage, are key elements to atherosclerotic plaque instability. ${ }^{3,4}$ Destabilization of plaque can lead to plaque rupture, with thrombus formation on the disrupted plaque surface and subsequent embolization of thrombus and/or plaque material into the distal vessels. Currently, MR imaging can accurately detect plaque components in the carotid arteries. ${ }^{5-7}$

Ischemic vascular brain disease, manifesting as brain infarcts and white matter lesions (WMLs), is highly prevalent in the elderly, often occurs subclinically, and is associated with an increased risk of adverse neurologic events. ${ }^{8,9}$ It is, however, unknown whether atherosclerotic plaque composition also relates to these vascular brain lesions in the general population. Morphologically, brain infarcts can be divided into lacunar infarcts and cortical infarcts. Whereas lacunar infarcts are associated with local occlusive disease of deep perforating arteries, ${ }^{10}$ cortical infarcts are primarily caused by thrombo-embolism from extracranial arteries or the heart. ${ }^{11}$ For WMLs, cerebral small-vessel disease is considered the main contributory factor. Therefore, we 
hypothesized that vulnerable carotid plaque components as assessed on MR imaging are associated with cortical infarcts rather than with lacunar infarcts or WMLs.

In 951 individuals from the population-based Rotterdam Study, ${ }^{12}$ we studied the association of carotid atherosclerotic plaque components, intraplaque hemorrhage, and lipid core, with markers of ischemic brain disease on MR imaging.

\section{MATERIALS AND METHODS \\ Study Population}

The current study was embedded in the Rotterdam Study, a prospective, population-based study among middle-aged and elderly participants ( $>95 \%$ of white descent), aimed at investigating determinants of various diseases among middle-aged and elderly individuals ( 45 years of age or older). ${ }^{12}$ All participants are invited at regular time intervals, every 3-4 years, to the research center for follow-up examinations, including carotid ultrasonography. ${ }^{13}$ From October 2007 onward, participants with carotid wall thickening (maximum thickness, $\geq 2.5 \mathrm{~mm}$ on sonography) in the left, right, or both carotid arteries were invited for MR imaging of the carotid arteries. Until August 2010, 7151 participants of the Rotterdam Study cohort had undergone ultrasonography and were still alive. In $26.3 \%$ of these participants $(n=1882)$, carotid wall thickening was present on sonography. We excluded individuals who had MR imaging contraindications $(n=43)$, had dementia ( $n=12)$, had physical immobility $(n=50)$, lived in nursing homes $(n=60)$, had moved outside the area $(n=54)$, or had undergone a carotid endarterectomy procedure $(n=4)$. Of the 1659 subjects eligible for carotid MR imaging, 1451 agreed to participate (response, $87.5 \%$ ). Due to physical inabilities (eg, back pain) or claustrophobia, imaging could not be performed or completed in 85 individuals (5.9\%), so, in total, 1366 participants underwent complete carotid MR imaging. The quality of all sequences in each carotid MR imaging was rated on a 5-point scale $(1=$ worst, 5 = best $) .{ }^{14}$ Scans were included in the analyses if the image quality was scored $\geq 3(n=1289,94.4 \%)$ in all carotid MR imaging sequences. Exclusion $(n=77)$ was mainly due to susceptibility artifacts from dental implants and motion artifacts.

For the current study, we selected participants with carotid MR imaging of sufficient quality who had also undergone an MR imaging examination of their brains as a part of the Rotterdam Scan Study, ${ }^{15}$ an ongoing study investigating age-related brain changes on MR imaging. Invitation to the Rotterdam Scan Study was independent of participation in the carotid MR imaging study. The objectives and design of the Rotterdam Scan Study have been described in detail elsewhere. ${ }^{16}$ Until August 2010, in total, 951 participants underwent complete MR imaging examinations of both carotid arteries and brain and were included in the current study. The median time interval between the brain and carotid MR imaging was 9 (25th-75th percentile, 2-21) months, with the brain MR imaging preceding the carotid MR imaging in $88.5 \%$ of the subjects.

The Rotterdam Study has been approved by the medical ethics committee according to the Population Study Act Rotterdam Study, executed by the Ministry of Health, Welfare and Sports of the Netherlands. Written informed consent was obtained from all participants.

\section{Carotid MR Imaging}

Imaging of the carotid arteries was performed on a $1.5 \mathrm{~T}$ scanner (Signa HD; GE Healthcare, Milwaukee, Wisconsin) with a bilateral phased array surface coil (Machnet, Eelde, the Netherlands). We planned the high-resolution MR imaging sequences so that the carotid plaques were imaged completely on both sides, with a previously described standardized protocol. ${ }^{17}$ First, both carotid bifurcations were identified by means of $2 \mathrm{D}$ time-of-flight $\mathrm{MR}$ angiography. Thereafter, high-resolution MR imaging sequences were planned to image the carotid bifurcations on both sides: a proton density (PD)-weighted FSE black-blood sequence; a PDweighted FSE black-blood sequence with an increased in-plane resolution; a PD-weighted EPI sequence; a T2WI EPI sequence; a 3D-T1WI gradient-echo sequence; and, finally, a 3D phased-contrast MR angiography. ${ }^{17}$

\section{Assessment of Plaque Composition on Carotid MR Imaging}

Carotid plaques were analyzed by a single observer with $>3$ years' experience in rating carotid MR imaging, as previously described. ${ }^{17}$ Analysis involved examination of both carotid arteries. The presence of atherosclerotic plaque was defined as carotid wall thickness of $\geq 2.0 \mathrm{~mm}$, and carotid plaque was quantified by measuring maximum carotid wall thickness in the PD-weighted FSE images and by calculation of luminal stenosis with the NASCET criteria. ${ }^{18}$ In all carotid arteries with atherosclerotic plaque on MR imaging ( $n=1752$ carotids in 951 participants), the presence or absence of 3 different plaque components (calcification, intraplaque hemorrhage, and lipid core) was assessed with criteria described elsewhere. ${ }^{17}$ For examples of assessment of vulnerable plaque components, see Fig 1.

\section{Brain MR Imaging}

Brain MR imaging, details of which are described elsewhere, ${ }^{16}$ was performed on the same $1.5 \mathrm{~T}$ scanner. In short, the imaging protocol included 3 high-resolution axial scans used for assessment of infarcts and white matter lesions (ie, a T1WI sequence, a PDweighted sequence, and a FLAIR sequence).

Lacunar and cortical infarcts were rated on FLAIR, PDweighted, and T1WI sequences by 1 of 5 trained raters according to criteria described previously. ${ }^{16}$ The presence of infarcts and location (left or right hemisphere) were recorded. WMLs and intracranial volume were segmented with a validated fully automated tissue-classification technique, ${ }^{19,20}$ and total WML volume was calculated by summing all WML voxels across the whole brain and per hemisphere. Quantification of WML volume was not possible in 41 brain scans because of susceptibility or motion artifacts. Therefore, 1670 of 1752 carotid arteries were used to study associations between carotid plaque characteristics and WML volumes. Readers who interpreted the brain MR imaging were blinded to the carotid MR imaging findings, and vice versa.

\section{Cardiovascular Risk Factors}

Cardiovascular covariates were ascertained with standard procedures as described previously. ${ }^{21}$ Risk factors used as covariates in the current study were hypertension (defined as grade 2 

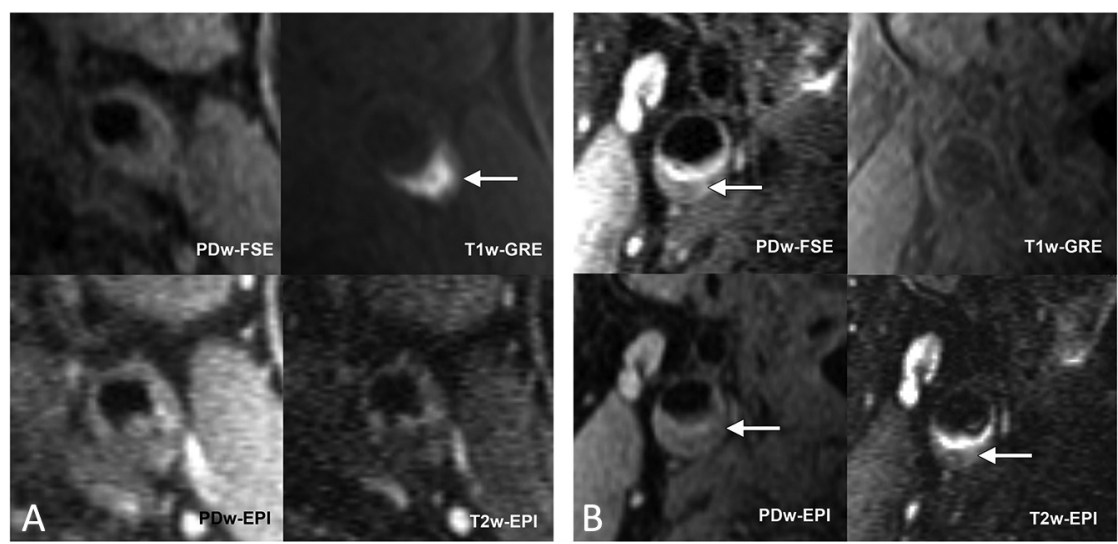

\section{Prevalent Stroke}

A history of stroke was based on the definition in the World Health Organization criteria, including a syndrome of rapidly developing symptoms, with an apparent vascular cause, of focal or global cerebral dysfunction lasting 24 hours or longer or leading to death. We assessed prevalent stroke at baseline during an interview and verified these data with medical records. ${ }^{23}$

\section{Statistical Analysis}

We assessed the relation of carotid

FIG 1. MR imaging of the carotid artery with atherosclerotic plaque containing vulnerable plaque components. Examples of the presence of intraplaque hemorrhage and lipid core in a carotid plaque. A, Axial images of the left carotid artery of a 72-year-old subject with carotid plaque formation in the carotid bifurcation. On the Tl-weighted gradient-echo image (T7w-GRE), the carotid plaque has a high signal intensity, identifying intraplaque hemorrhage (white arrow). $B$, Axial images of the right carotid artery of a 67-year-old subject with carotid plaque formation in the proximal internal carotid artery. There is no high signal intensity in the carotid plaque on the T1-weighted gradient-echo image. There is a region of low signal intensity in the plaque burden on both PD-weighted (PDW)-EPI and T2-weighted (T2w)-EPI images in the absence of high signal on the Tl-weighted gradient-echo image, identifying the presence of a lipid core (white arrows).

Table 1: Characteristics of the study population $(n=951)^{a}$

\begin{tabular}{lc}
\hline \multicolumn{1}{c}{ Characteristics } & $71.2 \pm 10.3$ \\
\hline Age (mean) (yr) & $444(46.7)$ \\
Women (No.) (\%) & \\
Cardiovascular risk factors (No.) (\%) & $489(51.4)$ \\
$\quad$ Hypertension, grade 22 & \\
$\quad$ Smoking & $474(49.8)$ \\
$\quad$ Past & $233(24.5)$ \\
$\quad$ Current & $142(14.9)$ \\
Diabetes mellitus & $307(32.3)$ \\
Hypercholesterolemia & \\
Cerebrovascular disease & $57(6.0)$ \\
History of clinical stroke & \\
MRI carotid characteristics & $306(32.2)$ \\
Intraplaque hemorrhage (No.) (\%) & $359(37.7)$ \\
Lipid core (No.) (\%) & $769(80.9)$ \\
Calcifications (No.) (\%) & $3.6 \pm 1.0$ \\
Maximum plaque thickness (mean) (mm) & $766(80.5)$ \\
Carotid stenosis of 0\%-29\% (No.) (\%) & $114(12.0)$ \\
Carotid stenosis of 30\%-49\% (No.) (\%) & $51(5.4)$ \\
Carotid stenosis of 50\%-69\% (No.) (\%) & $20(2.1)$ \\
Carotid stenosis of 70\%-99\% (No.) (\%) & \\
MRI brain characteristics & $62(6.5)$ \\
Cortical infarct on MRI (No.) (\%) & $125(13.1)$ \\
Lacunar infarct on MRI (No.) (\%) & $4.66(2.24-12.49)$ \\
WML volume (median) ${ }^{b}$ (interquartile & \\
$\quad$ range) (mL) & \\
\hline
\end{tabular}

${ }^{a}$ Categoric variables are presented as numbers. Continuous values are expressed as means. ${ }^{b}$ WML volume measurements were possible in 910 of 951 subjects because of susceptibility or motion artifacts.

according to European Society of Cardiology criteria $^{22}$ ), smoking (classified as current, past, and never), diabetes mellitus (defined as the use of blood glucose-lowering medication and/or a nonfasting serum glucose level of $11.1 \mathrm{mmol} / \mathrm{L}$ or higher and/or a fasting serum glucose level of $\geq 7 \mathrm{mmol} / \mathrm{L}$ $[\geq 126 \mathrm{mg} / \mathrm{dL}]$ ), and hypercholesterolemia (defined as a serum total cholesterol above $6.2 \mathrm{mmol} / \mathrm{L}$ [ $\geq 239 \mathrm{mg} / \mathrm{dL}]$ ). sis, each carotid plaque component was scored positive if present in 1 or both carotid arteries. For the artery-based analysis, only brain infarcts in the hemisphere ipsilateral to the examined carotid artery were taken into account. Associations between carotid plaque characteristics and brain infarcts were analyzed with logistic regression models, and the associations with WML volume were analyzed with linear regression models. WML volume was natural log-transformed because of skewness of the untransformed measure. For the analyses with WML volume as a dependent variable, persons with cortical infarcts were excluded to avoid misclassification from gliosis around the infarct area.

All analyses were adjusted for age, sex, and time interval between brain MR imaging and carotid MR imaging (model A). Associations with WML volumes were also adjusted for intracranial volume to take differences in head size into account. In addition, all analyses were adjusted for major cardiovascular risk factors (model B) and were adjusted for carotid luminal stenosis (model C). Additional stratified analyses were performed to assess effect modification by sex. Also, analyses were repeated after exclusion of individuals with a history of clinical stroke at baseline to compare associations with individuals with and without a history of stroke. To correct for within-person correlated carotid plaque data in the artery-based analyses, we followed the generalized estimation equation approach with an unstructured working correlation matrix. The unit in the analysis was the presence of ischemic brain disease in the left and/or right hemisphere. Each participant yielded a maximum of $2 \mathrm{U}$ in the dataset. In case of missing values for the cardiovascular risk factors, values were imputed by using the expectation-maximization method $(<1 \%$ of the data). Analyses were performed with SPSS for Windows, Version 22.0 (IBM, Armonk, New York). The $P$ value threshold used for significance was .05 . 
Table 2: Associations between carotid characteristics and cortical infarcts ${ }^{\mathrm{a}}$

\begin{tabular}{|c|c|c|c|c|c|c|c|c|c|c|c|c|}
\hline & \multicolumn{6}{|c|}{ Per Participant $(n=951)$} & \multicolumn{6}{|c|}{ Per Carotid Artery $(n=1752)$} \\
\hline & $\underset{A^{b}}{\text { Model }}$ & $\begin{array}{c}P \\
\text { Value }\end{array}$ & $\underset{B^{C}}{\text { Model }}$ & $\begin{array}{c}P \\
\text { Value }\end{array}$ & $\underset{C^{d}}{\text { Model }}$ & $\begin{array}{c}P \\
\text { Value }\end{array}$ & $\underset{A^{b}}{\text { Model }}$ & $\begin{array}{c}P \\
\text { Value }\end{array}$ & $\begin{array}{c}\text { Model } \\
B^{C}\end{array}$ & $\begin{array}{c}P \\
\text { Value }\end{array}$ & $\underset{C^{d}}{\text { Model }}$ & $\begin{array}{c}P \\
\text { Value }\end{array}$ \\
\hline \multicolumn{13}{|l|}{ Plaque components } \\
\hline Intraplaque hemorrhage & $2.0(1.2-3.5)$ & .012 & $1.9(1.1-3.3)$ & .019 & $1.7(1.0-3.2)$ & .059 & $2.0(1.1-3.6)$ & .033 & $1.9(1.0-3.6)$ & .052 & $1.2(0.6-2.4)$ & .633 \\
\hline Lipid core & $1.3(0.8-2.2)$ & .337 & $1.3(0.8-2.2)$ & .318 & $1.1(0.6-1.9)$ & .697 & $1.6(0.9-2.8)$ & .076 & $1.6(0.9-2.8)$ & .077 & $1.0(0.6-1.9)$ & .881 \\
\hline Calcification & $1.1(0.5-2.3)$ & .863 & $1.0(0.5-2.2)$ & .977 & $1.0(0.5-2.0)$ & 911 & $1.6(0.8-3.1)$ & .162 & $1.6(0.8-3.0)$ & .177 & $1.2(0.6-2.5)$ & .552 \\
\hline \multicolumn{13}{|l|}{ Measures of plaque size } \\
\hline $\begin{array}{l}\text { Maximum carotid wall } \\
\text { thickness }\end{array}$ & $1.3(1.1-1.6)$ & .006 & $1.3(1.1-1.6)$ & .009 & $1.2(1.0-1.6)$ & .090 & $1.6(1.3-1.9)$ & $<.001$ & $1.6(1.3-1.9)$ & $<.001$ & $0.8(0.6-1.2)$ & .320 \\
\hline$\geq 30 \%$ stenosis $^{f}$ & $1.9(1.1-3.3)$ & .033 & $1.8(1.0-3.2)$ & . 048 & NA & NA & $3.1(1.6-6.0)$ & .001 & $3.1(1.6-6.1)$ & .001 & NA & NA \\
\hline
\end{tabular}

Note:-NA indicates not applicable.

${ }^{a}$ Values represent odds ratios ( $95 \%$ confidence intervals).

${ }^{b}$ Model A is adjusted for age, sex, and time interval between brain MRI and carotid MRI.

'Model B is adjusted for age, sex, time interval between brain MRI and carotid MRI, and major cardiovascular risk factors (hypertension, smoking, diabetes mellitus, and hypercholesterolemia).

${ }^{\mathrm{d}}$ Model $\mathrm{C}$ is adjusted for age, sex, carotid stenosis (when appropriate), and time interval between brain MRI and carotid MRI.

e Odds ratio per millimeter increase.

${ }^{f}$ Odds ratio of carotid stenosis of $\geq 30 \%$.

\section{RESULTS}

Table 1 shows baseline variables for the 951 participants. Mean age was $71.2 \pm 10.3$ years, and $444(46.7 \%)$ were women. Intraplaque hemorrhage was present in 306 (32.2\%); lipid core, in 359 (37.7\%); and calcification, in 769 (80.9\%) participants. Cortical infarcts were present in 62 subjects $(6.5 \%)$, and lacunar infarcts, in 125 subjects (13.1\%). Median WML volume was $4.66 \mathrm{~mL}$ (interquartile range, $2.24-12.49)$. In 1752 carotid arteries with wall thickening, 57 cortical infarcts $(3.3 \%)$ and 85 lacunar infarcts $(4.9 \%)$ were present in the ipsilateral hemisphere.

Results of the multivariate adjusted analysis on the association between carotid plaque characteristics and cortical infarcts are presented in Table 2. The presence of intraplaque hemorrhage (OR, 1.9; 95\% CI, 1.1-3.3), maximum carotid wall thickness (OR per millimeter increase, 1.3; 95\% CI, 1.1-1.6), and carotid stenosis of $\geq 30 \%$ (OR, 1.8 ; 95\% CI, 1.0-3.2) was all significantly associated with cortical infarcts in the subject-based multivariate analysis. When the association between intraplaque hemorrhage and cortical infarcts was adjusted for carotid stenosis, the estimate barely changed (OR, 1.7; 95\% CI, 1.0-3.2; $P=.059$ ), though the association became borderline significant. When we excluded participants with a history of clinical stroke at baseline from the analyses $(n=57)$, the association between intraplaque hemorrhage and cortical infarcts became stronger (OR, 2.4; 95\% CI, 1.2-4.6; $P=.01$, model $\mathrm{B}$ ), whereas it did not change for maximum wall thickness (OR, 1.3; 95\% CI, 1.0-1.6; $P=.05)$ and became nonsignificant for $>30 \%$ stenosis $(\mathrm{OR}, 1.5 ; 0.7-3.0 ; P=$ $.29)$.

In the carotid-based analysis, intraplaque hemorrhage was also significantly associated with the presence of ipsilateral cortical infarcts (Table 2). Lipid core presence and plaque calcification were not associated with cortical infarcts in the subject-based analyses, though the association for the lipid core was borderline significant in the carotid-based analysis (OR, 1.6; 95\% CI, 0.92.8; $P=.08$ ) (Table 2). After additional adjustments for carotid luminal stenosis, the association for intraplaque hemorrhage was nonsignificant (OR, 1.2; 95\% CI, 0.6-2.4; $P=.633$ ).

In Table 3 , the relation between carotid characteristics and lacunar infarcts on MR imaging is shown. There were no associations between any of the carotid plaque characteristics and the presence of lacunar infarcts. These results did not change after additional adjustment for carotid stenosis or exclusion of persons with clinical stroke or when performing artery-based analysis.

The association between carotid characteristics and WML volume is shown in Table 4. Subjects with calcifications had significantly larger WML volume (regression estimate, 0.21; $95 \%$ CI, 0.05-0.38). The presence of intraplaque hemorrhage was borderline significantly associated with higher WML volume (regression estimate, $0.13 ; 95 \% \mathrm{CI},-0.01-0.28 ; P=.07$ ), but this was further attenuated when adjusting for cardiovascular risk factors $(P=.145)$. These results did not change after additional adjustment for carotid stenosis or exclusion of those with clinical stroke. Maximum carotid wall thickness was significantly associated with higher WML volume (regression estimate, 0.08 ; 95\% CI, $0.01-0.15 ; P=.032$ ) when adjusting for carotid stenosis. This result was not present without adjustments for carotid stenosis.

In a sensitivity analysis in 311 subjects, excluding subjects with a time interval of $>1$ month between carotid and brain MR imaging, the estimates of the associations did not change (On-line Tables 1 and 2).

Stratification for sex did not show significant interaction by sex in any of the analyses (data not shown).

\section{DISCUSSION}

In a large sample of the general population of predominantly white descent, we studied, with MR imaging, the association between vulnerable carotid plaque components and ischemic vascular brain disease. We found that carotid intraplaque hemorrhage was associated with the presence of cortical infarcts, but not with lacunar infarcts. Plaque calcification and carotid wall thickness but not vulnerable plaque components were related to higher WML volume.

One of the major strengths of our study is the large sample of middle-aged and elderly subjects from the general population. Compared with small clinical studies of patients with symptomatic brain ischemia, the use of a community-dwelling population provides information on associations between carotid plaque characteristics and ischemic brain disease that are less biased by the effects of medical intervention or lifestyle modifications. Furthermore, apart from participant-based analyses, we also assessed 
Table 3: Associations between carotid characteristics and lacunar infarcts ${ }^{a}$

\begin{tabular}{|c|c|c|c|c|c|c|c|c|c|c|c|c|}
\hline & \multicolumn{6}{|c|}{ Per Participant $(n=951)$} & \multicolumn{6}{|c|}{ Per Carotid Artery $(n=1752)$} \\
\hline & $\underset{A^{b}}{\text { Model }}$ & $\begin{array}{c}P \\
\text { Value }\end{array}$ & $\underset{B^{C}}{\text { Model }}$ & $\begin{array}{c}P \\
\text { Value }\end{array}$ & $\underset{C^{d}}{\text { Model }}$ & $\begin{array}{c}P \\
\text { Value }\end{array}$ & $\underset{A^{b}}{\text { Model }}$ & $\begin{array}{c}P \\
\text { Value }\end{array}$ & $\begin{array}{c}\text { Model } \\
B^{C}\end{array}$ & $\begin{array}{c}P \\
\text { Value }\end{array}$ & $\underset{C^{d}}{\text { Model }}$ & $\begin{array}{c}P \\
\text { Value }\end{array}$ \\
\hline \multicolumn{13}{|l|}{ Plaque components } \\
\hline Intraplaque hemorrhage & $1.0(0.6-1.4)$ & .922 & $1.0(0.6-1.5)$ & .855 & $1.1(0.7-1.7)$ & .562 & $1.1(0.6-1.7)$ & .412 & $1.1(0.7-1.9)$ & .464 & $1.1(0.6-1.8)$ & .856 \\
\hline Lipid core & $0.9(0.6-1.4)$ & .782 & $1.0(0.7-1.5)$ & .974 & $1.0(0.7-1.6)$ & .832 & $1.1(0.6-1.8)$ & .815 & $1.0(0.6-1.8)$ & .766 & $1.0(0.6-1.7)$ & .946 \\
\hline Calcification & $1.0(0.6-1.8)$ & .885 & $1.0(0.6-1.7)$ & .992 & $1.1(0.6-1.9)$ & .705 & $1.0(0.6-1.6)$ & .890 & $1.1(0.6-1.5)$ & .773 & $0.9(0.5-1.4)$ & .573 \\
\hline \multicolumn{13}{|l|}{ Measures of plaque size } \\
\hline $\begin{array}{l}\text { Maximum carotid wall } \\
\text { thickness }^{e}\end{array}$ & $1.0(0.8-1.2)$ & .815 & $1.0(0.8-1.1)$ & .605 & $1.1(0.9-1.4)$ & .307 & $1.1(0.9-1.3)$ & .581 & $1.0(0.8-1.2)$ & .771 & $1.0(0.8-1.4)$ & .825 \\
\hline$\geq 30 \%$ stenosis $^{f}$ & $0.7(0.4-1.2)$ & .168 & $0.7(0.4-1.2)$ & .166 & NA & NA & $0.8(0.4-1.6)$ & .582 & $0.8(0.4-1.5)$ & .450 & NA & NA \\
\hline
\end{tabular}

Note:-NA indicates not applicable.

${ }^{a}$ Values represent odds ratios ( $95 \%$ confidence intervals).

${ }^{b}$ Model $A$ is adjusted for age, sex, and time interval between brain MRI and carotid MRI.

'Model B is adjusted for age, sex, time interval between brain MRI and carotid MRI, and major cardiovascular risk factors (hypertension, smoking, diabetes mellitus, and hypercholesterolemia).

${ }^{\mathrm{d}}$ Model $\mathrm{C}$ is adjusted for age, sex, carotid stenosis (when appropriate), and time interval between brain MRI and carotid MRI.

e Odds ratio per millimeter increase.

${ }^{f}$ Odds ratio of carotid stenosis of $\geq 30 \%$.

Table 4: Associations between subjects' carotid plaque characteristics and WML load $(n=887)^{\mathrm{a}}$

\begin{tabular}{|c|c|c|c|c|c|c|}
\hline & Model $A^{b}$ & $P$ Value & Model Bc & $P$ Value & Model C ${ }^{d}$ & $P$ Value \\
\hline \multicolumn{7}{|l|}{ Plaque components } \\
\hline Intraplaque hemorrhage & $0.13(-0.01-0.28)$ & .070 & $0.11(-0.04-0.25)$ & .145 & $0.13(-0.02-0.27)$ & .083 \\
\hline Lipid core & $-0.09(-0.22-0.05)$ & .203 & $-0.06(-0.19-0.07)$ & .353 & $-0.08(-0.20-0.05)$ & .277 \\
\hline Calcification & $0.23(0.06-0.39)$ & .007 & $0.21(0.05-0.38)$ & .010 & $0.21(0.05-0.37)$ & .008 \\
\hline \multicolumn{7}{|l|}{ Measures of plaque size } \\
\hline Maximum carotid wall thickness ${ }^{\mathrm{e}}$ & $0.05(-0.02-0.12)$ & .131 & $0.04(-0.03-0.10)$ & .235 & $0.08(0.01-0.15)$ & .032 \\
\hline$\geq 30 \%$ stenosis & $-0.10(-0.26-0.07)$ & .241 & $-0.11(-0.27-0.05)$ & .181 & NA & NA \\
\hline
\end{tabular}

Note:-NA indicates not applicable.

${ }^{a}$ Values represent increase in white matter lesion load (natural log-transformed) (95\% confidence intervals) for the presence of plaque components, the presence of carotid stenosis of $\geq 30 \%$, or per-millimeter increase in maximum wall thickness.

${ }^{\mathrm{b}}$ Model $\mathrm{A}$ is adjusted for age, sex, and time interval between brain MRI and carotid MRI.

${ }^{c}$ Model B is adjusted for age, sex, time interval between brain MRI and carotid MRI, and major cardiovascular risk factors (hypertension, smoking, diabetes mellitus and hypercholesterolemia).

${ }^{\mathrm{d}}$ Model C is adjusted for age, sex, carotid stenosis (when appropriate), and time interval between brain MRI and carotid MRI.

e Regression coefficients per millimeter increase.

the relation between left/right carotid plaque and ipsilateral ischemic brain disease, enabling us to investigate vascular brain disease in the corresponding vascular territory.

A potential limitation of our study is that a lipid core is best detected with contrast-enhanced MR imaging. ${ }^{24}$ Although we did not administer a contrast agent in our population-based sample, the non-contrast-enhanced sequences we used were shown to have good accuracy in validation studies. ${ }^{5,6,14}$ Furthermore, we assessed the presence of hemorrhage and lipid core but did not measure the size of each plaque component, which may have affected our sensitivity to detect relevant associations. Another potential limitation is the time interval between brain MR imaging and carotid MR imaging, which was, on average, 9 months, with brain MR imaging preceding carotid MR imaging in $89 \%$ of participants. Thus, brain infarcts could potentially have preceded plaque abnormalities in time, influencing our results. However, because the main aim of our study was not risk prediction but investigating etiologic parallels between plaque vulnerability and stroke, we believe that this sequencing will not have seriously influenced our results. Also, adjustment for time interval and sensitivity analysis in subjects with short time intervals between carotid and brain MR imaging did not change our results. Last, our cross-sectional study design makes it difficult to interpret cause and effect of the associations found.

In our study, the presence of intraplaque hemorrhage was related to cortical infarcts, and this seemed primarily true for men.
A small cross-sectional study in patients with recent ischemic events and carotid stenosis found that the size of intraplaque hemorrhage and not the presence of a lipid core was associated with symptomatic plaques. ${ }^{25}$ On the other hand, in a prospective MR imaging study in subjects with 50\%-79\% carotid stenosis, both intraplaque hemorrhage and the size of the lipid core were related to the risk of a cerebrovascular event, albeit not the presence of a lipid core itself. ${ }^{1}$ In our study, we only found a borderline significant association between the presence of a lipid core and cortical infarcts, and only for the ipsilateral hemisphere. Thus, on the basis of our findings and those of others, it may be hypothesized that carotid intraplaque hemorrhage, rather than the presence of a lipid core, is a more relevant indicator of plaque vulnerability. However, other studies with a more sensitive measure of lipid core and assessing lipid core size should further explore the relation between lipid core and cortical infarcts.

The association we found between intraplaque hemorrhage and the presence of cortical infarcts is in line with the presumed etiology of cortical infarcts, which are primarily regarded as being caused by large-vessel disease. In contrast, lesions in subcortical brain regions, such as lacunar infarcts and white matter lesions, are considered the sequelae of cerebral small-vessel disease. ${ }^{26}$ In our study, none of the vulnerable plaque characteristics-intraplaque hemorrhage and lipid core- or measures of plaque size were associated with the presence of lacunar infarcts, supporting 
extracranial carotid embolic disease not being an important factor in the development of lacunar infarcts.

We did not find important differences between the participant-based analyses and the analyses restricted to the ipsilateral hemisphere of each carotid artery, though the degree of carotid stenosis was a confounder for the association between intraplaque hemorrhage and ipsilateral cortical infarcts. Previously, a relationship between plaque burden expressed as severity of stenosis and the presence of intraplaque hemorrhage has been demonstrated. ${ }^{27}$ Intraplaque hemorrhage instead of the severity of stenosis must be considered as the link between atherosclerotic disease and brain infarcts. The predictive power of intraplaque hemorrhage versus stenosis should be evaluated in future studies. In addition, atherosclerosis in one vessel bed is known to reflect atherosclerotic activity in other vascular regions. ${ }^{28}$ Thus, carotid plaques are not per se only related to ischemic disease in the ipsilateral vascular territory. If we extended this concept of atherosclerosis as a generalized disease, intraplaque hemorrhage could be regarded as a focal manifestation of an underlying systemic disease process that includes several vulnerable lesions. ${ }^{29}$

In the ongoing discussion on the pathogenesis of WMLs, the most generally accepted concept is that they reflect a process of ischemic demyelination, predominantly with small-vessel disease as the etiologic factor. ${ }^{30}$ There is, however, also some evidence that large-vessel disease may contribute to the development of WMLs, ${ }^{31}$ especially via embolic events from unstable carotid plaques (ie, those with intraplaque hemorrhage). ${ }^{32}$ Although we found a borderline significant relation between carotid intraplaque hemorrhage and WML volume, this association attenuated when adjusting for cardiovascular risk factors. This finding may suggest that there is a shared etiology for atherosclerosis and the development of WMLs, rather than a direct causal connection between intraplaque hemorrhage and WML development.

In the current literature, all available data on this topic are from small studies in clinical patients, with 2 thoughts being put forward. Altaf et $\mathrm{al}^{32}$ found, in 187 symptomatic subjects, an association between carotid intraplaque hemorrhage and WML volume, supporting the large-vessel disease and unstable plaque concept. Kwee et $\mathrm{al}^{33}$ found, in 50 patients with symptomatic carotid stenosis, that carotid plaque burden was significantly associated with WML severity but that there was no causal relationship between carotid plaque vulnerability and the occurrence of WMLs. In contrast, Patterson et $\mathrm{al}^{34}$ found MR imaging plaque morphology (ie, plaque size, the presence of intraplaque hemorrhage, or lipid core) in 80 carotid arteries to be not independently predictive of the severity of WMLs, refuting the large-vessel disease and unstable plaque concept. Our results suggest no significant relation between intraplaque hemorrhage or lipid component and WML volume; therefore, our results contribute to this discussion in that they do not support the hypothesis of a role of vulnerable large-vessel atherosclerotic plaque in the development of WMLs. In the current study, carotid plaque calcification is associated with WMLs, also demonstrated in a previous CT-based study by Bos et al. ${ }^{35}$ This is possibly the association between luminal stenosis and brain MR imaging markers of ischemia rather than vulnerable plaque, because previous studies have suggested that for a given volume of plaque, the greater the proportion of calcification, the more "stable" the plaque is. ${ }^{36,37}$

\section{CONCLUSIONS}

The presence of carotid intraplaque hemorrhage and measures of carotid plaque size, as defined with MR imaging, are associated with MR imaging-defined cortical infarcts, but not with lacunar infarcts. Plaque instability does not seem to play a role in WML development.

Disclosures: Quirijn J.A. van den Bouwhuijsen—RELATED: Grant: Netherlands Heart Foundation, Comments: grants 2006B206 and 2009B044.* Benjamin F.J. VerhaarenRELATED: Grant: Dutch Heart Foundation, Comments: grant No. 2009B102.* Wiro J. Niessen-UNRELATED: Consultancy: Quantib BV, Comments: I am advisor (chief scientific officer) of Quantib BV*; Stock/Stock Options: Quantib BV, Comments: I am cofounder and shareholder of Quantib BV. Gabriel P. Krestin-UNRELATED: Consultancy: Bracco*; Grants/Grants Pending: Siemens, Bayer, GE Healthcare, Bracco*. *Money paid to the institution.

\section{REFERENCES}

1. Takaya N, Yuan C, Chu B, et al. Association between carotid plaque characteristics and subsequent ischemic cerebrovascular events: a prospective assessment with MRI-initial results. Stroke 2006;37: 818-23 CrossRef Medline

2. Saam T, Hatsukami TS, Takaya N, et al. The vulnerable, or high-risk, atherosclerotic plaque: noninvasive MR imaging for characterization and assessment. Radiology 2007;244:64-77 CrossRef Medline

3. Stary HC, Chandler AB, Dinsmore RE, et al. A definition of advanced types of atherosclerotic lesions and a histological classification of atherosclerosis: a report from the Committee on Vascular Lesions of the Council on Arteriosclerosis, American Heart Association. Circulation 1995;92:1355-74 CrossRef Medline

4. Redgrave JN, Lovett JK, Gallagher PJ, et al. Histological assessment of 526 symptomatic carotid plaques in relation to the nature and timing of ischemic symptoms: the Oxford plaque study. Circulation 2006;113:2320-28 CrossRef Medline

5. Cappendijk VC, Cleutjens KB, Kessels AG, et al. Assessment of human atherosclerotic carotid plaque components with multisequence MR imaging: initial experience. Radiology 2005;234:487-92 CrossRef Medline

6. Saam T, Ferguson MS, Yarnykh VL, et al. Quantitative evaluation of carotid plaque composition by in vivo MRI. Arterioscler Thromb Vasc Biol 2005;25:234-39 Medline

7. Moody AR. Magnetic resonance direct thrombus imaging. J Thromb Haemost 2003;1:1403-09 CrossRef Medline

8. Vermeer SE, Hollander M, van Dijk EJ, et al; Rotterdam Scan Study. Silent brain infarcts and white matter lesions increase stroke risk in the general population: the Rotterdam Scan Study. Stroke 2003;34: 1126-29 CrossRef Medline

9. van Dijk EJ, Prins ND, Vermeer SE, et al. Frequency of white matter lesions and silent lacunar infarcts. J Neural Transm Suppl 2002: 25-39 Medline

10. Fisher CM. Lacunar strokes and infarcts: a review. Neurology 1982; 32:871-76 CrossRef Medline

11. Warlow C, Sudlow C, Dennis M, et al. Stroke. Lancet 2003;362: 1211-24 CrossRef Medline

12. Hofman A, Darwish Murad S, van Duijn CM, et al. The Rotterdam Study: 2014 objectives and design update. Eur J Epidemiol 2013;28: 889-926 CrossRef Medline

13. Iglesias del Sol A, Bots ML, Grobbee DE, et al. Carotid intima-media thickness at different sites: relation to incident myocardial infarction-the Rotterdam Study. Eur Heart J 2002;23:934-40 CrossRef Medline

14. Yuan C, Mitsumori LM, Ferguson MS, et al. In vivo accuracy of multispectral magnetic resonance imaging for identifying lipid-rich 
necrotic cores and intraplaque hemorrhage in advanced human carotid plaques. Circulation 2001;104:2051-56 CrossRef Medline

15. Ikram MA, van der Lugt A, Niessen WJ, et al. The Rotterdam Scan Study: design update 2016 and main findings. Eur J Epidemiol 2015; 30:1299-315 CrossRef Medline

16. Vernooij MW, van der Lugt A, Ikram MA, et al. Prevalence and risk factors of cerebral microbleeds: the Rotterdam Scan Study. Neurology 2008;70:1208-14 CrossRef Medline

17. van den Bouwhuijsen QJ, Vernooij MW, Hofman A, et al. Determinants of magnetic resonance imaging detected carotid plaque components: the Rotterdam Study. Eur Heart J 2012;33:221-29 CrossRef Medline

18. North American Symptomatic Carotid Endarterectomy Trial: methods, patient characteristics, and progress. Stroke 1991;22: 711-20 CrossRef Medline

19. Vrooman HA, Cocosco CA, van der Lijn F, et al. Multi-spectral brain tissue segmentation using automatically trained k-nearest-neighbor classification. Neuroimage 2007;37:71-81 CrossRef Medline

20. de Boer R, Vrooman HA, van der Lijn F, et al. White matter lesion extension to automatic brain tissue segmentation on MRI. Neuroimage 2009;45:1151-61 CrossRef Medline

21. Vliegenthart R, Oudkerk M, Hofman A, et al. Coronary calcification improves cardiovascular risk prediction in the elderly. Circulation 2005;112:572-77 CrossRef Medline

22. European Society of Hypertension-European Society of Cardiology Guidelines Committee. 2003 European Society of Hypertension-European Society of Cardiology guidelines for the management of arterial hypertension. J Hypertens 2003;21:1011-53 CrossRef Medline

23. Wieberdink RG, van Schie MC, Koudstaal PJ, et al. High von Willebrand factor levels increase the risk of stroke: the Rotterdam study. Stroke 2010;41:2151-56 Medline

24. Cai J, Hatsukami TS, Ferguson MS, et al. In vivo quantitative measurement of intact fibrous cap and lipid-rich necrotic core size in atherosclerotic carotid plaque: comparison of high-resolution, contrast-enhanced magnetic resonance imaging and histology. Circulation 2005;112:3437-44 CrossRef Medline

25. Saam T, Cai J, Ma L, et al. Comparison of symptomatic and asymptomatic atherosclerotic carotid plaque features with in vivo MR imaging. Radiology 2006;240:464-72 CrossRef Medline
26. Pantoni L. Cerebral small vessel disease: from pathogenesis and clinical characteristics to therapeutic challenges. Lancet Neurol 2010;9:689-701 CrossRef Medline

27. Selwaness M, Hameeteman R, Van 't Klooster R, et al. Determinants of carotid atherosclerotic plaque burden in a stroke-free population. Atherosclerosis 2016;255:186-92 CrossRef Medline

28. Odink AE, van der Lugt A, Hofman A, et al. Association between calcification in the coronary arteries, aortic arch and carotid arteries: the Rotterdam study. Atherosclerosis 2007;193:408-13 CrossRef Medline

29. Lutgens E, van Suylen RJ, Faber BC, et al. Atherosclerotic plaque rupture: local or systemic process? Arterioscler Thromb Vasc Biol 2003;23:2123-30 CrossRef Medline

30. Pantoni L, Garcia JH. Pathogenesis of leukoaraiosis: a review. Stroke 1997;28:652-59 CrossRef Medline

31. de Leeuw FE, de Groot JC, Bots ML, et al. Carotid atherosclerosis and cerebral white matter lesions in a population based magnetic resonance imaging study. J Neurol 2000;247:291-96 CrossRef Medline

32. Altaf N, Morgan PS, Moody A, et al. Brain white matter hyperintensities are associated with carotid intraplaque hemorrhage. Radiology 2008;248:202-09 CrossRef Medline

33. Kwee RM, Hofman PA, Gronenschild EH, et al. Association between carotid plaque characteristics and cerebral white matter lesions: one-year follow-up study by MRI. PLoS One 2011;6:e17070 CrossRef Medline

34. Patterson AJ, UK-Im JM, Tang TY, et al. Association between white matter ischaemia and carotid plaque morphology as defined by high-resolution in vivo MRI. Eur J Vasc Endovasc Surg 2009;38: 149-54 CrossRef Medline

35. Bos D, Ikram MA, Elias-Smale SE, et al. Calcification in major vessel beds relates to vascular brain disease. Arterioscler Thromb Vasc Biol 2011;31:2331-37 CrossRef Medline

36. Kwee RM. Systematic review on the association between calcification in carotid plaques and clinical ischemic symptoms. J Vasc Surg 2010;51:1015-25 CrossRef Medline

37. Gupta A, Baradaran H, Kamel H, et al. Evaluation of computed tomography angiography plaque thickness measurements in highgrade carotid artery stenosis. Stroke 2014;45:740-45 CrossRef Medline 